\title{
Bilateral Chylothorax Secondary to Retrosternal Goitre: a Case Report and Review of the Literature
}

\author{
Ruth Patricia Cusak ${ }^{1}$, Jane McCarthy ${ }^{2}$, Terence Markham O'Connor ${ }^{1}$ \\ ${ }^{1}$ Department of Respiratory Medicine, Mercy University Hospital, Cork, Ireland \\ ${ }^{2}$ Department of Gastroenterology, Mercy University Hospital, Cork, Ireland
}

Received: $14 / 01 / 2016$

Accepted: $14 / 02 / 2016$

Published: $23 / 03 / 2016$

How to cite this article: Cusack RP, McCarthy J, O'Connor TM. Bilateral chylothorax secondary to retrosternal goitre: a case report and review of the literature. EJCRIM 2016;3:doi:10.12890/2016_000388

Conflicts of Interests: The Authors declare that there are no competing interests.

This article is licensed under a Commons Attribution Non-Commercial 4.0 License

\section{ABSTRACT}

Chylothorax is characterized by an accumulation of lymphatic fluid in the pleural cavity due to damage to the thoracic duct. The aetiology can be traumatic or non-traumatic. Goitre is a rare cause of chylothorax with only eight cases previously described in the literature including only one case causing a bilateral chylothorax. This report describes a patient with bilateral chylothorax secondary to substernal goitre, which was successfully treated, and discusses this very rare case in light of the available literature.

\section{LEARNING POINTS}

- Pleural ultrasound and aspiration is important in bilateral pleural effusions unresponsive to diuretic treatment, or of dubious origin.

- The aetiology, diagnosis and treatment of a chylothorax are described.

- Goitre can cause chylothorax by damaging the thoracic duct.

\section{KEYWORDS}

Pleural effusion; chylothorax; goitre

\section{CASE REPORT}

A 70-year-old woman was referred to the emergency department by her general practitioner with a year long history of increasing dyspnoea. She stated her dyspnoea had gradually worsened over 1 year. On admission she was dyspnoeic on minimal exertion. Her background included a 30-year history of goitre with inspiratory stridor, asthma and obesity. She was on no regular medications. She was a non-drinker and a non-smoker.

On examination the patient was dyspnoeic with reduced air entry bilaterally with stony dullness. Her oxygen saturations were $92 \%$ on room air. Her abdomen was distended with a fluid thrill and she had pitting oedema up to her mid shin. She had an obvious multinodular substernal goitre (Fig. 1) and Pemberton's sign was negative.

Chest $x$-ray revealed bilateral pleural effusions. She had mildly deranged liver function tests with aspartate aminotransferase of 57 and gamma-glutamyl transpeptidase of 52 . She was mildly hypoalbuminaemic with an albumin of $31 \mathrm{~g} / \mathrm{l}$. Her thyroid function tests were normal. Viral hepatitis screen was negative.

The patient was admitted with presumed decompensated heart failure and treated with diuretics. Peripheral and abdominal oedema improved; however, she remained dyspnoeic with bilateral pleural effusions. Echocardiography showed normal heart function and liver 
ultrasound revealed liver cirrhosis causing her abdominal ascites and lower limb oedema.

Pleural ultrasound performed at the patient's bedside showed bilateral pleural effusions. The right effusion appeared moderate and complex, with no loculations. Pleural aspirate revealed a milky brown fluid consistent with a chylothorax. A chest drain was inserted and 1.2 litres of fluid was drained (Fig. 2). Analysis of the fluid revealed a pH of 7.53, triglycerides $19.3 \mathrm{~g} / \mathrm{dl}$ and predominantly lymphocytic fluid.

Bronchoscopy showed circumferential $5 \mathrm{~cm}$ tracheal narrowing distal to the cords with no invasion. A thyroid ultrasound and computerised tomography (CT) of the neck/thorax confirmed a large multinodular goitre with thoracic duct compression and bilateral pleural effusions (Figs. 3 and 4). The patient had a total thyroidectomy performed with repair of her thoracic duct and resolution of her bilateral pleural effusions.

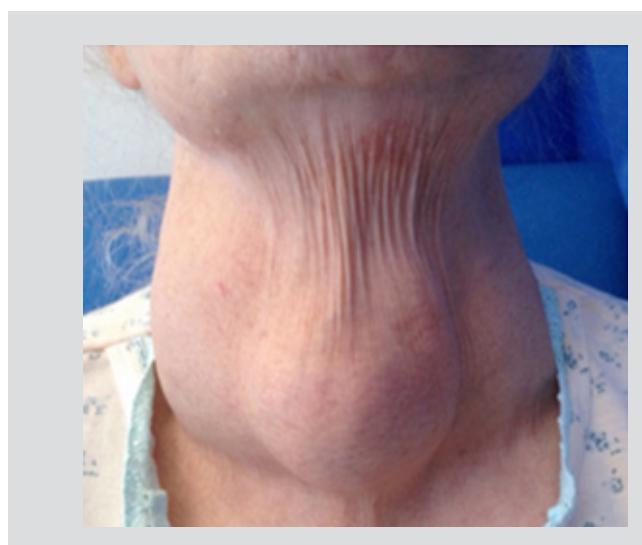

Figure 1: Multinodular goitre.

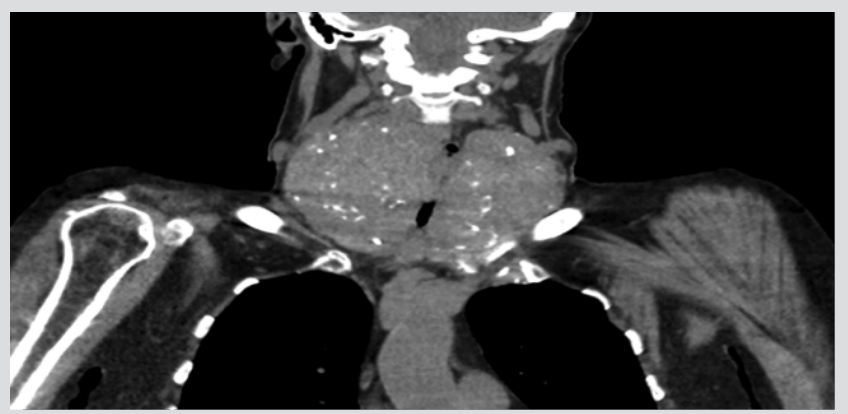

Figure 3: Computerised tomographic images showing multinodular goitre with substernal extension.

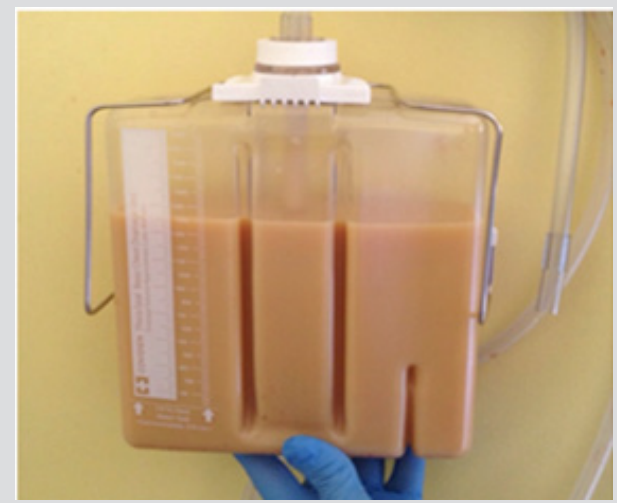

Figure 2: Chest drain underwater seal bottle showing the milky brown fluid collection of a chylothorax secondary to a retrosternal goitre.

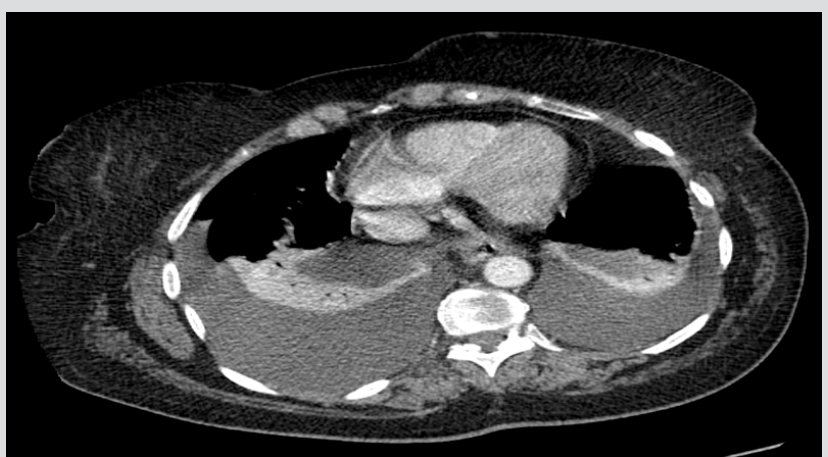

Figure 4: Computerised tomographic images showing bilateral pleural effusions

\section{DISCUSSION}

Chylothorax is the collection of lymphatic fluid in the pleural space caused by damage to the thoracic duct. Chylothorax may be unilateral, either right (50\%) or left sided (33\%), or bilateral (16.6\%) and is dependent on the site of the thoracic duct leak ${ }^{[1]}$. Damage to the thoracic duct above $T 5$ results in a left-sided effusion, while damage to the duct below T5 results in a right-sided effusion ${ }^{[2]}$.

The aetiology can be traumatic or non-traumatic ${ }^{[3]}$. Disruption of the thoracic duct anywhere along its course can cause a chylothorax. Surgical procedures such as oesophagectomy, cardiac surgery and lung resection carry the greatest traumatic risk. Malignancy is the leading cause of non-traumatic chylothorax. Lymphoma, chronic lymphocytic leukaemia and metastatic cancer have all been associated with this condition previously. Less common causes include sarcoidosis, tuberculosis, constrictive pericarditis and Castleman's disease. Goitre is a rare cause of chylothorax with only eight cases previously described in the literature, including only one case causing a bilateral chylothorax ${ }^{[4]}$.

Patients with chylothorax usually present with symptoms and signs secondary to the development of a pleural effusion: cough, dyspnoea 
and chest pain. Pleural fluid is typically milky white in appearance with lymphocyte predominance. Fluid analysis shows it is an exudative effusion which is triglyceride rich (>11 g/dl), and the presence of chylomicrons confirms the diagnosis ${ }^{[1]}$. The cholesterol level in a chylothorax is usually less than $5.18 \mathrm{mmol} / \mathrm{l}$ and distinguishes a chylothorax from a cholesterol effusion.

CT of the thorax, abdomen and pelvis can be performed to identify any lymphadenopathy, or cardiac or pulmonary cause for the chylothorax. It will also identify any retrosternal goitre. CT lymphangiography can be used to identify the exact site of the leak. If constrictive pericarditis is suspected, an echocardiograph should be performed.

Conservative treatment initially involves the insertion of a pleural drain if the patient is symptomatic and to ensure lung expansion. A low fat diet with medium chain triglycerides can reduce the volume of chyle in the thoracic duct, slowing reaccumulation and allowing the thoracic duct to heal. Surgical therapy is indicated when conservative therapy fails or chyle flow persists ${ }^{[1]}$. Thoracic duct ligation can be performed by thoracotomy.

As goitre causing a chylothorax is so rare, there are no treatment guidelines, and thus we are currently guided by previous case reports. In our case, a total thyroidectomy with thoracic duct ligation was performed as our patient had significant compression symptoms from her goitre: stridor, dyspnoea and bilateral chylothorax. This resulted in resolution of her bilateral pleural effusions.

\section{CONCLUSIONS}

Bilateral chylothorax secondary to a goitre is extremely rare; however, it should be suspected in patients with a substernal goitre. In our case, the patient had total thyroidectomy and repair of her thoracic duct, with resolution of her bilateral effusions post operatively.

\section{REFERENCES}

1. McGrath EE, Blades Z, Anderson P. Chylothorax: aetiology, diagnosis and therapeutic options. Resp Med 2010;104:1-8.

2. Bessone LN, Ferguson TB, Burford TH. Chylothorax. Ann Thorac Surg 1971;12:527-550.

3. Doerr CH, Allen MS, Nichols FC, Ryu JH. Etiology of chylothorax in 203 patients. Mayo Clin Proc 2005;80:867-870.

4. Simmgen M, Newlands ES, Southcott BM, Vigushin DM. Bilateral chylothorax due to retrosternal goiter in a patient with non-Hodgkin's lymphoma. Med Oncol 2001;18:153157. 